\title{
Rhizosphere bacteria degrade auxin to promote root growth
}

\author{
Baoyuan $Q u^{1,2,3, *}$ \\ 1 State Key Laboratory of Plant Genomics, Institute of Genetics and Developmental Biology, Innovation Academy for Seed Design, \\ Chinese Academy of Sciences, Beijing 100101, China \\ 2 CAS Center for Excellence in Biotic Interactions, University of Chinese Academy of Sciences, Beijing 100049, China \\ 3 CAS-JIC Centre of Excellence for Plant and Microbial Sciences, Institute of Genetics and Developmental Biology, Chinese Academy of \\ Sciences, Beijing 100101, China \\ (C) Higher Education Press 2021
}

Dynamic microbial communities exist both within and around plant tissues (Bulgarelli et al., 2012; Lundberg et al., 2012). Complex interactions among microorganisms, as well as between microorganisms and plants, are governed by a wide range of chemical signals and influence plant growth directly and indirectly (Lebeis et al., 2015; Durán et al., 2018; Finkel et al., 2019; Huang et al., 2019). Antagonistic interactions among microorganisms shape the plant microbiota and protect plants against pathogens. For example, Pseudomonas piscium isolated from the wheat Yannong 19 head microbiome inhibits the growth and virulence of the plant pathogenic fungus Fusarium graminearum by modulating the activity of its histone acetyltransferase (Chen et al., 2018). Moreover, a Flavobacterium cultivated from the root microbiota of wilt-resistant tomato can suppress Ralstonia solanacearum in susceptible tomato (Kwak et al., 2018). In addition to such direct antagonistic interactions, microorganisms inhibit the secretion of chemical signals produced by other microorganisms, which alters plant-microorganism signaling. Auxin is one of the phytohormones that has dual function on plant growth-promotion at low level but inhibition at high level (Evans et al., 1994). Indole-3-acetic acid (IAA), the main form of auxin, synthesized not only by plant, but also by plant growth promotion rhizobacteria (PGPR) (Vejan et al., 2016; Cherif-Silini et al., 2019). However, how plants can maintain stereotypic growth in surrounding with PGPR that produce high level of auxin remained unknown. In a recent study, Finkel and colleagues found out the answer of the question, they reported that a single bacterial genus could degrade IAA released by the rhizosphere microbiome, thus maintaining plant root growth in the presence of a complex microbiome, which sheds light on how plants can successfully inhabit the complex soil environment (Finkel et al., 2020).

The authors used a deconstructed synthetic community to represent the complex rhizosphere microbiome and investigated how interactions among microorganisms shape plant growth. They inoculated Arabidopsis seedlings with a 185-member synthetic bacterial community on agar plates, subjected the plates to 16 abiotic stress conditions, and assessed the bacterial community in agar, the rhizosphere and roots. Subsequently, they divided the full community into four phylogenetically related modules (A, B, C and D) by calculating the pairwise correlation in relative abundance across all samples. Module A contained mainly Gammaproteobacteria prefer to enriched in the substrate than in the seedlings and B contained mainly Firmicutes with no significant enrichment trend; modules $C$ and $D$ were composed mainly of Alphaproteobacteria and Actinobacteria, respectively, both of those were enriched in seedlings. To test the effects of each module or their combinations on primary root length, the authors inoculated Arabidopsis seedlings with each module singly, or with each pairwise combination of communities (AB, AC, $A D, B C, B D$ and $C D$ ). Although inoculation with module $C$ or $D$ led to significant root growth inhibition (RGI) of Arabidopsis seedlings, inoculation with module A or B did not. Screening resulted in the identification of 34 taxonomically diverse strains distributed within all four modules that induced an RGI phenotype, but this could not explain how the full community in the presence of module A did not trigger RGI.

Notably, Finkel et al. (2020) observed that the RGI induced by modules C or D was attenuated in the presence of module A, indicating that module A contained specific strains that could modulate RGI and maintain stereotypic root development. Screening

Received January 15, 2021; Revised March 9, 2021; Accepted March 25, 2021

* Corresponding author

E-mail address: byqu@genetics.ac.cn (B. Qu) 
all non-RGI bacteria in module A for their ability to attenuate the RGI induced by representative strains from the four modules led to the observation that that all bacteria that belonged to the genus Variovorax from module A could revert the RGI phenotype induced by MF224 and CL28, from module $C$ and module D, respectively. A similar result was obtained when two representative Variovorax species were screened against all RGI-induced strains: these species attenuated 13 out of 18 RGI-induced strains tested. Similar results were obtained in other culture media, including sterile soil, and in the presence of additional Variovorax species not included within the community of 185 species. These findings can be used to promote plant root growth in the field, which might support efforts to enhance adaptation to environmental stresses, such as nutrient deficiency.

Plants respond rapidly to environment challenges, often via transcriptional reprogramming. The transcription of marker genes for phosphate $(\mathrm{Pi})$ starvation can be induced by inoculation with a bacterial community under Pi starvation (Castrillo et al., 2017). Finkel et al. (2020) attempted to identify the mechanisms that underlie how Variovorax suppresses RGI, by analyzing the transcriptomes of Arabidopsis roots in the presence or absence of Variovorax. They observed that RGI induced a large proportion of genes with functions related to the root apex. Notably, the genes induced in RGI seedlings included GH3.2, which is responsible for the conversion of excess IAA to IAA-AA, and 12 auxin-responsive genes (Takase et al., 2004; Uchida et al., 2018).

Plant root growth is tightly regulated by plant hormones, especially auxin and ethylene. Auxin can be synthesized and degraded by plant-associated microbiota, which affect plant growth, including root growth. The authors indeed observed that Variovorax could suppress RGI induced by IAA and ethylene and degraded auxin in substrates, suggesting that RGI suppression by Variovorax may involve interference with bacterially induced auxin signaling.

The authors investigated the mechanisms involved in RGI attenuation in further detail. They identified 947 genes unique to Variovorax that were assigned to hot spots based on their physically contiguous presence on the chromosome. One of these, hot spot 33, shared sequence similarity to the iac operon, which functions in auxin degradation (Leveau and Gerards, 2008). The genes in hot spot 33 were significantly upregulated by coculture of Variovorax and RGI-inducing strains. Furthermore, genetically engineered Variovorax bacteria that contained the hot spot 33 fragment acquired the ability to degrade IAA, and reverted RGI induced by IAA and other bacterial strains. By contrast, mutant Variovorax strains in which hot spot 33 was deleted lost the ability to degrade IAA and suppress RGI. This finding indicates that members of the Variovorax genus contain genes that can induce auxin degradation, which enables Variovorax to promote stereotypic root development in the context of a phylogenetically diverse microbiome (Fig.1).

Plants grow in a complex soil environment that contains a diverse microbial community, and interactions among microbes and among microbes and plants that are guided by chemicals play an important role in plant health. Moreover, these complex interactions are extremely important for agricultural productivity. Finkel and his colleagues (2020) demonstrated that bacteria from
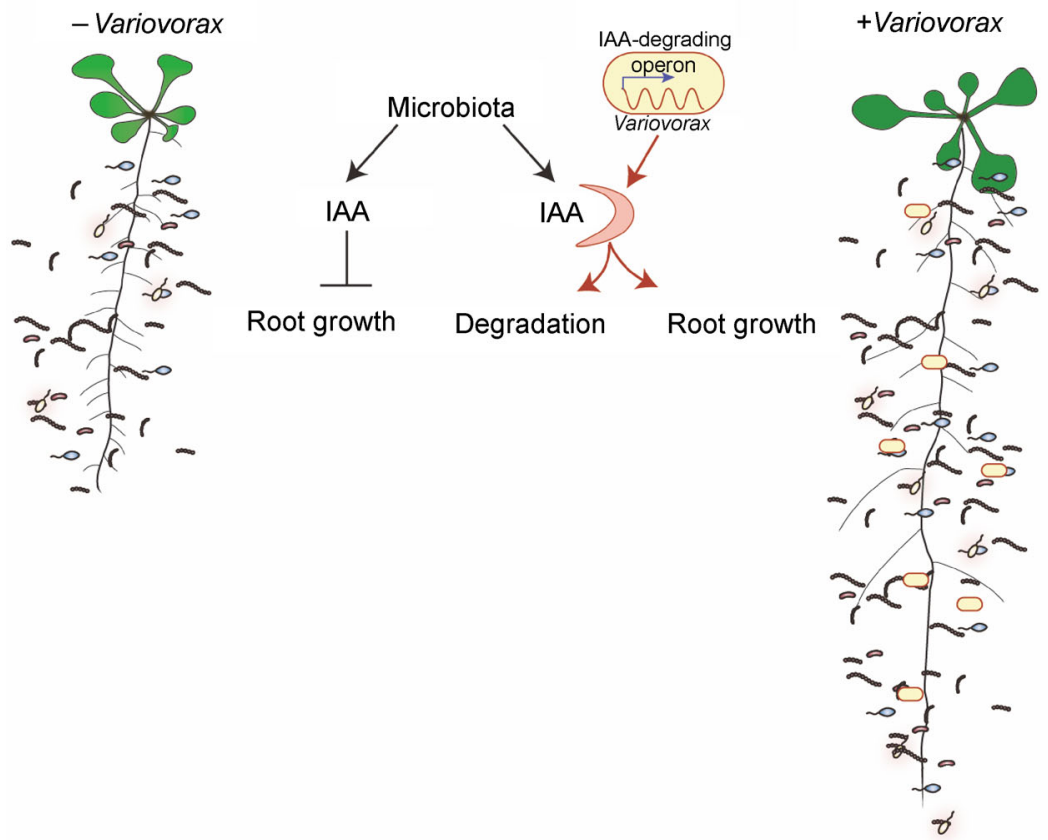

Fig. 1 Members of the bacterial genus Variovorax degrade microbiota-derived auxin to promote plant root growth. Following incubation with a Variovorax drop-out synthetic community, the growth of Arabidopsis primary roots is inhibited by the high IAA concentration generated by the root microbiota. In the presence of Variovorax, the IAA-degrading operon synthesizes enzymes that degrade IAA released by the root microbiota, which then releases roots from growth inhibition. 
the Variovorax genus contain an auxin-degrading operon that allows them to degrade excess IAA generated by the microbiota surrounding plant roots and thereby enables Variovorax bacteria to counteract plant RGI induced by the root microbiota. Many questions remain to be addressed, including how to interpret this effect of Variovorax species on IAA and root growth given that these species are depleted in the rhizosphere compared with bulk soil. In addition, the precise functions of Variovorax species in IAA degradation remain to be elucidated, as do whether abiotic stresses, such as nutrient deficiency, that modify plant root architecture via plant hormone levels influence the abundance of Variovorax species and whether the level of members of the Variovorax genus could be manipulated in the rhizosphere to promote root growth and thereby induce tolerance to distinct stresses.

\section{Acknowledgments}

This work was supported by grants from the Strategic Priority Research Program of the Chinese Academy of Sciences (Precision Seed Design and Breeding, XDA24020104), the Key Research Program of Frontier Sciences of the Chinese Academy of Sciences (QYZDBSSW-SMC021), the National Natural Science Foundation of China (31772400, 31761143017, 31801945, 31701997).

\section{Declaration of Interests}

The author declare no competing interests.

\section{References}

Bulgarelli, D., Rott, M., Schlaeppi, K., Ver Loren van Themaat, E., Ahmadinejad, N., Assenza, F., Rauf, P., Huettel, B., Reinhardt, R., Schmelzer, E., Peplies, J., Gloeckner, F.O., Amann, R., Eickhorst, T., Schulze-Lefert, P., 2012. Revealing structure and assembly cues for Arabidopsis rootinhabiting bacterial microbiota. Nature 488, 91-95.

Castrillo, G., Teixeira, P.J., Paredes, S.H., Law, T.F., de Lorenzo, L., Feltcher, M.E., Finkel, O.M., Breakfield, N.W., Mieczkowski, P., Jones, C.D., Paz-Ares, J., Dangl, J.L., 2017. Root microbiota drive direct integration of phosphate stress and immunity. Nature 543, 513-518.

Chen, Y., Wang, J., Yang, N., Wen, Z., Sun, X., Chai, Y., Ma, Z., 2018. Wheat microbiome bacteria can reduce virulence of a plant pathogenic fungus by altering histone acetylation. Nature Communications 9, 3429-3442.

Cherif-Silini, H., Thissera, B., Bouket, A.C., Saadaoui, N., Silini, A., Eshelli, M., Alenezi, F.N., Vallat, A., Luptakova, L., Yahiaoui, B., Cherrad, S., Vacher, S., Rateb, M.E., Belbahri, L., 2019. Durum wheat stress tolerance induced by endophyte Pantoea agglomerans with genes contributing to plant functions and secondary metabolite arsenal. International Journal of Molecular Sciences 20, 3989-4024.

Durán, P., Thiergart, T., Garrido-Oter, R., Agler, M., Kemen, E., Schulze-Lefert, P., Hacquard, S., 2018. Microbial interkingdom interactions in roots promote Arabidopsis survival. Cell 175, 973-983.e14.

Evans, M.L., Ishikawa, H., Estelle, M.A., 1994. Responses of Arabidopsis roots to auxin studied with high temporal resolution: comparison of wildtype and auxin-response mutants. Planta 194, 215-222

Finkel, O.M., Salas-González, I., Castrillo, G., Conway, J.M., Law, T.F., Teixeira, P.J.P.L., Wilson, E.D., Fitzpatrick, C.R., Jones, C.D., Dangl, J.L., 2020. A single bacterial genus maintains root growth in a complex microbiome. Nature 587, 103-108.

Finkel, O.M., Salas-González, I., Castrillo, G., Spaepen, S., Law, T.F., Teixeira, P.J.P.L., Jones, C.D., Dangl, J.L., 2019. The effects of soil phosphorus content on plant microbiota are driven by the plant phosphate starvation response. PLoS Biology 17, e3000534.

Huang, A.C., Jiang, T., Liu, Y.X., Bai, Y.C., Reed, J., Qu, B., Goossens, A., Nützmann, H.W., Bai, Y., Osbourn, A., 2019. A specialized metabolic network selectively modulates Arabidopsis root microbiota. Science 364, eaau6389.

Kwak, M.J., Kong, H.G., Choi, K., Kwon, S.K., Song, J.Y., Lee, J., Lee, P.A., Choi, S.Y., Seo, M., Lee, H.J., Jung, E.J., Park, H., Roy, N., Kim, H., Lee, M.M., Rubin, E.M., Lee, S.W., Kim, J.F., 2018. Author Correction: Rhizosphere microbiome structure alters to enable wilt resistance in tomato. Nature Biotechnology 36, 1117.

Lebeis, S.L., Paredes, S.H., Lundberg, D.S., Breakfield, N., Gehring, J., McDonald, M., Malfatti, S., Glavina del Rio, T., Jones, C.D., Tringe, S.G., Dangl, J.L., 2015. PLANT MICROBIOME. Salicylic acid modulates colonization of the root microbiome by specific bacterial taxa. Science 349 , 860-864.

Leveau, J.H., Gerards, S., 2008. Discovery of a bacterial gene cluster for catabolism of the plant hormone indole 3-acetic acid. FEMS Microbiology Ecology 65, 238-250.

Lundberg, D.S., Lebeis, S.L., Paredes, S.H., Yourstone, S., Gehring, J., Malfatti, S., Tremblay, J., Engelbrektson, A., Kunin, V., Del Rio, T.G., Edgar, R.C., Eickhorst, T., Ley, R.E., Hugenholtz, P., Tringe, S.G., Dangl, J.L., 2012. Defining the core Arabidopsis thaliana root microbiome. Nature 488, 86-90.

Takase, T., Nakazawa, M., Ishikawa, A., Kawashima, M., Ichikawa, T., Takahashi, N., Shimada, H., Manabe, K., Matsui, M., 2004. ydk1-D, an auxin-responsive GH3 mutant that is involved in hypocotyl and root elongation. Plant Journal 37, 471-483.

Uchida, N., Takahashi, K., Iwasaki, R., Yamada, R., Yoshimura, M., Endo, T.A., Kimura, S., Zhang, H., Nomoto, M., Tada, Y., Kinoshita, T., Itami, K., 
Hagihara, S., Torii, K.U., 2018. Chemical hijacking of auxin signaling with an engineered auxin-TIR1 pair. Nature Chemical Biology 14, 299305.

Vejan, P., Abdullah, R., Khadiran, T., Ismail, S., Nasrulhaq Boyce, A., 2016. Role of plant growth promoting rhizobacteria in agricultural sustainability-A review. Molecules (Basel, Switzerland) 21, 573-589. 